\title{
La información al paciente sobre los servicios profesionales farmacéuticos asistenciales de farmacia comunitaria
}

\author{
Vicente J. Baixauli Fernández', Fernando Abellán-García Sánchez², Ana Molinero Crespo ${ }^{3}$, Rosa Prats Más4, \\ Javier Plaza Zamora ${ }^{5}$, Leyre Gaztelurrutia Lavesa ${ }^{6}$, Sara Bellver Beltrán ${ }^{7}$, María del Mar Arranz Esteban ${ }^{8}$, \\ José Luis García-Espona Pancorbo ${ }^{9}$, Javier Cremades Alcaraz ${ }^{10}$, Noelia Amador Fernández ${ }^{11}$
}

1. Doctor en Farmacia por la Universidad CEU-Cardenal Herrera. Responsable servicios profesionales de SEFAC. Farmacéutico comunitario en Mislata (Valencia). 2. Doctor en Medicina Legal y Abogado de Derecho Sanitario Asesores. 3. Doctora en Farmacia por la Universidad Complutense. Responsable del Área científica de SEFAC. Farmacéutica comunitaria en Fuenlabrada (Madrid). 4. Doctora en Farmacia por la Universidad de Granada. Coordinadora grupo de trabajo de HTA y riesgo vascular. Farmacéutica comunitaria en Denia (Alicante). 5. Doctor en Farmacia por la Universidad de Murcia. Coordinador del Grupo de trabajo de Respiratorio y tabaquismo de SEFAC. Farmacéutico comunitario en Mazarrón (Murcia). 6. Coordinadora del Área de Tabaquismo del Grupo Respiratorio y tabaquismo de SEFAC. Farmacéutica comunitaria en Barakaldo (Bizkaia). 7. Coordinadora de la subcomisión de Revisión del Uso de los Medicamentos (RUM) de SEFAC. Farmacéutica comunitaria en Onda (Castellón). 8. Miembro de la subcomisión de Revisión del Uso de los Medicamentos (RUM) de SEFAC. Farmacéutica comunitaria en Brighton (Reino Unido). 9. Miembro de la subcomisión de Revisión del Uso de los Medicamentos (RUM) de SEFAC. Farmacéutico comunitario en Huéscar (Granada). 10. Doctor en Farmacia por la Universidad Miguel Hernández (Elche). Miembro de la subcomisión de Revisión del Uso de los Medicamentos (RUM) de SEFAC. Farmacéutico comunitario en Aspe (Alicante). 11. Farmacéutica. Departamento técnico de SEFAC.

\section{PALABRAS CLAVE}

farmacia comunitaria, servicios farmacéuticos, información al paciente, consentimiento informado, farmacéutico comunitario

\section{ABREVIATURAS}

SPFA: Servicio profesional farmacéutico asistencial de la farmacia comunitaria

\section{KEYWORDS}

Community pharmacy, pharmaceutical services, consumer health information, informed consent, community pharmacist

\section{RESUMEN}

La prestación de servicios profesionales farmacéuticos asistenciales (SPFA) al paciente desde la farmacia comunitaria ha de realizarse observando una serie de garantías sanitarias y legales previas que aseguren los derechos del paciente. Toda actuación en el ámbito de la salud de un paciente necesita su consentimiento libre y voluntario una vez que haya recibido la información adecuada. Esta información que, como regla general, se proporcionará verbalmente (salvo en los casos de procedimientos invasivos, que son los que entrañan riesgos más graves), ha de ser veraz e incluir como mínimo la finalidad y la naturaleza de cada intervención, sus riesgos y sus consecuencias, y se debe comunicar al paciente de forma comprensible y adaptada a sus necesidades, de forma que le ayude a tomar decisiones de acuerdo con su propia y libre voluntad. De este modo, para que el paciente consienta que se le preste un SPFA ha de recibir previamente una información específica sobre dicho servicio. Aunque esta información se podría proporcionar solo verbalmente, al ser los SPFA servicios novedosos con los que el paciente no está familiarizado, es recomendable proporcionarla también por escrito ya que esta vía permite garantizar que se comunica de forma uniforme, comprensible, evitando equivocaciones y olvidos, y además el paciente tendrá más tiempo para estudiarla en su casa. Este artículo analiza la información mínima que se debe proporcionar a los pacientes y usuarios en la prestación de los SPFA. Además, muestra como ejemplo tanto la información que se proporciona al paciente en cinco de los SPFA que gestiona la aplicación SEFAC e_XPERT ${ }^{\circledR}$, como un modelo de consentimiento informado válido para dichos servicios y que incluye el resto de información necesaria que también se debe comunicar previamente al paciente.

\section{Information to patients on professional pharmaceutical care services of} Community Pharmacy

\section{ABSTRACT}

The provision of professional pharmaceutical care services (SPFA) to the patient from the community pharmacy must be carried out by observing a series of previous health and legal guarantees that ensure the rights of the patient. Any action in the area of a patient's health requires his or her free and voluntary consent once he or she had received the appropriate information. This information will, as a general rule, be provided orally (except in the case of invasive procedures, which are the ones with the most serious risks), must be truthful and include at least the purpose and nature of each intervention, its risks and its consequences, and must be communicated to the patient in a way that is understandable and adapted to his or her needs, in a way that helps you make decisions according to your own free will. Thus, in order for the patient to consent to the provision of an SPFA, he must first receive specific information about that service. Although this information could be provided only verbally, as SPFAs are novel services with which the patient is not familiar, it's advisable to provide it also in writing, as this way ensures that it communicates uniformly, comprehensively, avoiding mistakes and forgetfulness, and also that the patient will have more time to study it at home. This article discusses the minimum information to be provided to patients and users in the provision of SPFA. In addition, it shows, as an example, the information provided to the patient in five of the SPFA that manage the SEFAC e_XPERT ${ }^{\circledR}$ application, as well as an informed consent model which includes all other necessary information which must also be communicated to patient.

Financiación: ninguna.

Conflicto de intereses: ninguno.

Cite este artículo como: Baixauli VJ, Abellán-Garcia F, Molinero A, Prats R, Plaza J, Gaztelurrutia L, Bellver S, Arranz MM, Garcia-Espona JL, Cremades J, Amador N. La información al paciente sobre los servicios profesionales farmacéuticos asistenciales de farmacia comunitaria. Farmacéuticos Comunitarios. 2019 Sep 30;11(3):22-41. doi:10.5672/FC.2173-9218. (2019/Vol11).003.05

Correspondencia: Vicente J. Baixauli Fernández (vtejbaixauli@sefac.org).

ISSN 1885-8619 @SEFAC (Sociedad Española de Farmacia Familiar y Comunitaria). Todos los derechos reservados. 
Los servicios profesionales farmacéuticos asistenciales de farmacia comunitaria (SPFA) se definen como aquellas actividades sanitarias prestadas desde la farmacia comunitaria por un farmacéutico que emplea sus competencias profesionales para la prevención de la enfermedad y la mejora tanto de la salud de la población como la de los destinatarios de los medicamentos y productos sanitarios, desempeñando un papel activo en la optimización del proceso de uso y de los resultados de los tratamientos. Dichas actividades, alineadas con los objetivos generales del sistema sanitario, tienen entidad propia, con definición, fines, procedimientos y sistemas de documentación, que permiten su evaluación y retribución, garantizando su universalidad, continuidad y sostenibilidad (1).

La Ley 41/2002, básica reguladora de la autonomía del paciente y de derechos y obligaciones en materia de información y documentación clínica (2), como su nombre indica, tiene por objeto la regulación de los derechos y obligaciones de los pacientes, usuarios y profesionales, asi como de los centros $y$ servicios sanitarios, públicos y privados, en materia de autonomía del paciente y de información y documentación clínica.

Los principios básicos de esta ley son los que justifican las consideraciones legales previas que es necesario tener en cuenta en la farmacia comunitaria para la prestación de los SPFA. En este sentido, previamente a la prestación de cualquier SPFA en la farmacia comunitaria, el farmacéutico ha de conocer la información que se le debe proporcionar al paciente para que este dé su consentimiento.

La Ley de autonomía del paciente establece que toda actuación en el ámbito de la salud de un paciente necesita el consentimiento libre $y$ voluntario del afectado, una vez que, recibida la información prevista (....) haya valorado las opciones propias del caso. Los SPFA son actividades que conllevan actuaciones en el ámbito de la salud de un paciente, por lo que están sometidos a esta ley y, por tanto, es requisito previo imprescindible que el paciente libre y voluntariamente decida si consiente o no que se le preste un SPFA determinado, al haberle informado sobre dicho servicio. Este requisito tiene además sentido, como se dijo más arriba, si se tiene en cuenta que algunos SPFA todavía no son conocidos ampliamente por la población, por ser servicios novedosos que en su mayoría todavía no han sido regulados por una normativa, ni están financiados por el Sistema Nacional de Salud, o no han sido prestados tradicionalmente en la farmacia $\mathrm{y}$, además, que requieren para su prestación de la colaboración del paciente (3).

Algunos farmacéuticos podrían pensar que la información/publicidad escrita (folletos...) o visual (anuncios, carteles...) de un determinado SPFA en la farmacia comunitaria es suficiente para cumplir con la obligación de información al paciente. Sin embargo, es conveniente conocer los requisitos éticos y legales que debe cumplir esta información en relación con los siguientes aspectos:

\section{Finalidad}

El objetivo de esta información destinada al paciente es que le ayudará a tomar decisiones de acuerdo con su propia y libre voluntad.

\section{Veracidad}

La información que se le proporcione al paciente ha de ser verdadera.

\section{Información mínima}

La información mínima que se debe proporcionar al paciente ha de incluir la finalidad y la naturaleza de cada intervención, sus riesgos y sus consecuencias. También es importante y recomendable incluir en la información destinada al paciente, que la prestación de un SPFA no sustituye a la visita a su médico cuando esta sea precisa (por ejemplo, cuando empeore su salud).

Información sobre protección de datos, precio del SPFA, consentimiento informado y posible revocación de dicho consentimiento

Al margen de la información clínica que incluyen estas hojas de información al paciente sobre cada SPFA, es importante tener en cuenta que, previamente a que el paciente o usuario de los SPFA otorgue su consentimiento informado para su realización, de acuerdo con el Reglamento Euro- peo de Protección de Datos (RGPD) (4) y la Ley orgánica de protección de datos personales y garantía de los derechos digitales (LOPD-GDD) (5), también es necesario informarle sobre los derechos de acceso, rectificación, supresión, limitación, oposición y portabilidad (derechos ARCO-POL) sobre los datos personales y sanitarios que se recaben en cada SPFA. Además, conforme exige la Ley de defensa de los consumidores y usuarios (6), es obligado informar del precio del SPFA y, por último, según la Ley de autonomía del paciente, también hay que informarle de que si diera su consentimiento informado siempre tiene la posibilidad de revocarlo.

Sin embargo, en las hojas de información sobre cada SPFA que gestiona la aplicación informática SEFAC e_XPERT ${ }^{\circledR}$, desarrollada por la Sociedad Española de Farmacia Familiar y Comunitaria (SEFAC), y que se incluyen como ejemplo en este artículo (anexos 1-5), no está contemplada la citada información sobre protección de datos personales, el precio del SPFA y la revocación del consentimiento, porque la misma, aparte de que se transmita verbalmente por el farmacéutico, se encuentra consignada en el documento de consentimiento informado (anexos 6-8). De esta forma se separa la información de carácter clínico específica de cada SPFA del resto de información de carácter más formal.

\section{Necesidad de una entrevista personal}

Como se establece en el Código Ético de la Farmacia Comunitaria (7), el ofrecimiento de un SPF exige también que, previamente, se haya mantenido una entrevista en la farmacia con el paciente para explicarle pormenorizadamente que su contratación es absolutamente voluntaria, en qué consiste el servicio, cuáles son los logros $y$ ventajas que se esperan conseguir, los riesgos posibles y los compromisos que debe asumir, asi como la conveniencia, en su caso, de suscribir un documento escrito de consentimiento informado, en cuyo supuesto se le permitirá un margen de tiempo suficiente para que lo pueda analizar $y$ valorar con tranquilidad. También debe informársele de que se respetará su decisión de darse de baja en cualquier momento que lo desee, sin que 
ello implique desatención ni ningún tipo de rechazo por el farmacéutico para seguir prestándole otros servicios distintos.

\section{Lenguaje utilizado}

De acuerdo con su finalidad la información se debe comunicar al paciente de forma comprensible $y$ adecuada a sus necesidades. Este requisito, en la práctica, implica la necesidad de proporcionarles la información en castellano y/o en los idiomas cooficiales de su territorio que entiendan y conozcan, así como en el caso de pacientes extranjeros de hacer todo lo posible para que la información sea comprendida. Por otro lado, la información ha de ser comprensible, utilizando un lenguaje sin tecnicismos y expresiones complejas. La información que se ofrece como ejemplo en este artículo debería adaptarse y/o explicarse en caso necesario con el fin de que el paciente la comprenda y le permita decidir.

\section{Vía de comunicación}

Si bien la Ley 41/2002 (2) indica que como regla general se proporcionará verbalmente dejando constancia en la historia clinica..., también se puede comunicar, complementariamente, de forma escrita por las ventajas que dicha vía ofrece.

\section{Destinatario}

La información sobre el SPFA se le debe dar tanto al paciente como a las personas vinculadas a él por razones familiares o de hecho, en la medida que el paciente lo permita de manera expresa o tácita (2), lo que ampararía la presencia del cuidador en caso necesario cuando esta se proporcione. Únicamente en caso de que el estado físico o psíquico del paciente no le permitiera hacerse cargo de su situación o no quisiera recibirla o en los supuestos de menores de edad no maduros, podría no proporcionarse la información directamente a este. En caso de que el paciente no quisiera recibir esta información, este tiene derecho a que se respete su voluntad de no ser informado, si bien dicha decisión debe quedar registrada en su historia clínica. En el ámbito de la farmacia comunitaria como el farmacéutico no tiene acceso a esta decisión del paciente debería quedar registrada en el correspondiente consentimiento informado del SPFA.

\section{Lugar}

La Ley de autonomía del paciente (2) también establece el derecho a la intimidad, que engloba tanto a la confidencialidad de los datos como al secreto profesional. Toda persona tiene derecho a que se respete el carácter confidencial de los datos referentes a su salud, y a que nadie pueda acceder a ellos sin previa autorización amparada por la Ley. De ahí que en la farmacia donde se presten SPFA para garantizar la intimidad del paciente al proporcionar esta información respecto a otras personas, se debe disponer de una zona de atención personalizada (ZAP) que garantice la confidencialidad de la información proporcionada y facilite que el paciente pueda preguntar sus dudas.

\section{Validez temporal}

En coherencia con los anteriores apartados, la información proporcionada al paciente ha de ser actualizada según los cambios que se produzcan en relación con la prestación del servicio $\mathrm{y}$, en especial, con los nuevos datos aportados por el conocimiento científico y la evidencia generada como consecuencia de su prestación.

En resumen, antes de prestar un SPFA el usuario ha de conocer la información mínima que le permita decidir si desea recibirlo. Por ello es conveniente disponer de esta información en la farmacia para cada SPFA. Además, hay que tener en cuenta que la información va dirigida a una persona y que habrá que adaptarla y explicarla si es necesario, incluso en otro idioma. Conforme los SPFA vayan implementándose en la farmacia comunitaria cada vez será menos frecuente que el usuario requiera de esta información para decidir solicitar la prestación de un determinado SPFA.

\section{Referencias bibliográficas}

1. Foro AF FC. Servicios Profesionales Farmacéuticos asistenciales; definiciones y clasificación. Disponible en: https://www. sefac.org/sites/default/files/sefac2010/private/documentos_sefac/documentos/6\%C2\%BA\%20comunicado\%20F0R0\%20 AF\%20FC_Servicios_Profesionales_Farmac\%C3\%A9uticos_Asistenciales.pdf

2. Ley $41 / 2002$, de 14 de noviembre, básica reguladora de la autonomía del paciente y de derechos y obligaciones en materia de información y documentación clínica. Boletín oficial del Estado, n²74, (15/11/2002). Disponible en: https://www.boe.es/buscar/act.php?id=BOE-A-2002-22188

3. Baixauli VJ, Abellán-García F. El consentimiento y el tratamiento de los datos sanitarios del paciente en la prestación y realización de estudios de investigación de servicios profesionales farmacéuticos asistenciales. Farmacéuticos Comunitarios. 2019 Mar 29; 11(1):24-28. doi:10.5672/FC.21739218.(2019/Vol11).001.05

4. Reglamento (UE) 2016/679 del Parlamento Europeo y del Consejo, de 27 de abril de 2016 relativo a la protección de las personas físicas en lo que respecta al tratamiento de datos personales y a la libre circulación de estos datos y por el que se deroga la Directiva 95/46/CE (Reglamento general de protección de datos). Diario Oficial de la Unión Europea, nº119, (4-5-2016). Disponible en: https://eur-lex.europa. eu/legal-content/ES/TXT/PDF/?uri=0$\mathrm{J}: L: 2016: 119$ :FULLCtfrom=ES

5. Ley orgánica 3/2018, de 5 de diciembre, de protección de datos personales y garantía de los derechos digitales. Boletín oficial del Estado, n²94, (6-12-2018). Disponible en: https://www.boe.es/boe/dias/2018/12/06/ pdfs/BOE-A-2018-16673.pdf

6. Real Decreto Legislativo 1/2007, que aprueba el texto refundido de la Ley General para la Defensa de los Consumidores y Usuarios. Boletín oficial del Estado, nº 287 (30-11-2007). Disponible el texto consolidado en: https://www.boe.es/buscar/pdf/2007/ BOE-A-2007-20555-consolidado.pdf

7. Comisión de Bioética de SEFAC. Código ético para la dispensación de medicamentos de uso humano no sujetos a prescripción médica, a través de sitios webs de las farmacias comunitarias. Madrid: Tecnología y Ediciones del Conocimiento (Edittec); 2014. ISBN: 97884-617-0039-4. Disponible en: https:// www.campussefac.org/product/codigo-etico-dispensacion-medicamentos-uso-humano-no-sujetos-prescripcion-medica-traves 


\author{
ANEXO 1. Hoja de información al paciente del Servicio de cribado y \\ abordaje de la EPOC de SEFAC e_XPERT ${ }^{\circledR}$
}

\title{
EXPLICACIÓN DEL SERVICIO DE CRIBADO Y ABORDAJE DE LA EPOC
}

De conformidad con la Ley 41/2002 de autonomía del paciente, esta explicación tiene como objetivo informarle sobre el servicio de cribado y abordaje que se presta en esta farmacia.

\section{FINALIDAD DE ESTE SERVICIO}

Detectar si puede padecer una Enfermedad Pulmonar Obstructiva Crónica (EPOC).

Si usted padece de EPOC, mejorar el control de su enfermedad a través del seguimiento y supervisión de los fármacos que toma. Asesorarle e implicarle en el control del tratamiento de la EPOC.

Este servicio profesional farmacéutico asistencial no sustituye a la visita a su médico cuando esta sea preciso (por ejemplo, cuando empeore su salud).

\section{¿CUÁNDO ESTÁ INDICADO?}

El cribado de la EPOC (realización de una prueba o test para detectar si una persona puede padecer EPOC o no), está indicado para cualquier paciente mayor de 35-40 años fumador o exfumador (con un consumo acumulado de tabaco de más de 100 cigarrillos en toda su vida) con síntomas respiratorios (tos con mucosidad, falta de aire en las actividades diarias...) pero todavía no diagnosticado de EPOC que acude a esta farmacia. Mientras que el abordaje de la EPOC está dirigido a aquellos pacientes que ya están diagnosticados de EPOC y son usuarios de inhaladores de forma crónica y pueden beneficiarse de un control y seguimiento de su medicación en la farmacia.

\section{¿EN QUÉ CONSISTE?}

El servicio de cribado y abordaje de la EPOC es un servicio profesional farmacéutico asistencial no gratuito que consiste en la realización por el farmacéutico junto con usted (o su cuidador en caso necesario) de la detección de la EPOC y de la comprobación de los medicamentos que utiliza para tratar la EPOC. El cribado de EPOC puede realizarse en la farmacia comunitaria, a través de distintos métodos de medición validados en función de sus características y necesidades, mientras que la comprobación de los medicamentos que utiliza para tratar la EPOC que usted utiliza, se realiza a través del asesoramiento farmacéutico (manejo de sus inhaladores, adherencia a los tratamientos, control de efectividad y seguridad de la medicación), el seguimiento del número de situaciones de agravamiento de los síntomas, la percepción de su calidad de vida y la colaboración con el resto de profesionales sanitarios. 


\section{¿DÓNDE SE REALIZA Y CUÁNTO TIEMPO REQUIERE?}

Este servicio profesional se lleva a cabo mediante una entrevista en una zona de atención personalizada de la farmacia que garantiza la confidencialidad. La información recabada está sometida al secreto profesional, salvo que usted decida que se comparta con su médico u otro profesional sanitario. La duración dependerá de si está usted diagnosticado de EPOC o no, si no lo está y quiere realizar en ese momento el cribaje, la visita puede durar 10 minutos. Si ya está diagnosticado de EPOC la primera visita en este caso podría durar entre 15 y 30 minutos y el resto de ellas alrededor de 10-15 minutos.

Una vez terminada la visita le entregaremos un informe del servicio acompañado -si procede- de una hoja de información para usted y de un documento de comunicación con otros profesionales sanitarios.

\section{¿QUÉ SE NECESITA?}

Pedir una cita en la farmacia para que se le preste el servicio.

Para el abordaje de la EPOC, se requiere que el día que se le vaya a prestar traiga a la farmacia todos los medicamentos y productos que utilice para mantener su salud (plantas medicinales, remedios, etc.), así como la hoja o receta donde figure la prescripción de dichos tratamientos.

Para la realización del cribado de la EPOC hay que realizar un cuestionario COPD PS, que determinará un posible riesgo de padecer la enfermedad, que puede complementarse con una espirometría forzada, es decir, un estudio rápido e indoloro que permite medir la cantidad de aire que aspira por la boca y pueden retener los pulmones, así como la velocidad de las inhalaciones y exhalaciones durante la respiración. Para realizar esta prueba se utiliza un aparato llamado espirómetro, siendo habitualmente el más utilizado en la farmacia el COPD-6. El espirómetro deberá aplicárselo sobre su boca y espirar el aire con fuerza durante unos segundos. Para que dicha espirometría sea válida es necesario que antes de realizarla tenga en cuenta las siguientes instrucciones: no haber tomado bebidas excitantes (café, cola, cacao, té...) en las 6 horas previas, no fumar como mínimo en las 3 ó 4 horas previas, no haber comido grandes cantidades 2-3 horas antes, no haber realizado ejercicio físico intenso 30 minutos antes, estar en reposo durante 5 minutos antes de la medida y no llevar ropa ajustada que dificulte la respiración. En caso de no haber seguido estas instrucciones dígaselo al farmacéutico que le preste este servicio.

\section{¿QUÉ BENEFICIOS SE PUEDEN ESPERAR?}

El abordaje de la EPOC puede mejorar el conocimiento, el cumplimiento, la adherencia y el proceso de uso de los inhaladores que usa para su salud, así como mejorar la calidad de vida asociada a la EPOC, disminuir la cantidad y gravedad de los ingresos hospitalarios y las exacerbaciones de la EPOC.

El cribado de la EPOC le permitirá detectar si usted puede padecer EPOC y en caso de que su médico le diagnostique, podrá comenzar con el tratamiento, mejorar los síntomas respiratorios, prevenir la progresión de la enfermedad y mejorar el estado de salud y la calidad de vida. 


\section{¿QUÉ RIESGOS SE PUEDEN ESPERAR?}

En el caso del cribado de la EPOC no se le realizará la espirometría si por sus características físicas o psíquicas es incapaz de realizar la prueba, ni si está embarazada. Aunque en condiciones normales no existe ninguna contraindicación formal para la realización de una espirometría durante el embarazo, existen ciertos casos en los que el riesgo supera con mucho a los posibles beneficios de realizarla, por lo que en estos casos, las espirometrías se realizarán en centros especializados. Por tanto, si usted presenta alguna de estas características o está embarazada dígaselo al farmacéutico que le preste este servicio. Por lo demás, este servicio no tiene en principio ningún riesgo para usted, ya que cuando la recomendación del farmacéutico consista en la supresión, cambio o variación de dosis de un medicamento de prescripción médica, deberá ponerse en conocimiento de su médico para que to valide su realización antes de hacer efectiva la modificación.

\section{¿QUÉ CONSECUENCIAS PUEDE TENER SU REALIZACIÓN?}

La mejora del uso de los inhaladores que utilice.

La mejora de la salud que comportaría ajustar bien la medicación tras la revisión que se le lleve a cabo, una disminución en la frecuencia de las exacerbaciones e intensidad de las mismas.

La detección de una posible EPOC haciendo posible el comenzar de forma temprana el tratamiento de la enfermedad, lo que mejorará la evolución de la EPOC y su calidad de vida. 


\author{
ANEXO 2. Hojas de información al paciente del Servicio de cesación \\ tabáquica de SEFAC $\mathrm{e}_{-} \mathrm{XPERT}{ }^{\circledR}$
}

\title{
EXPLICACIÓN DEL SERVICIO DE CESACION TABAQUICA
}

De conformidad con la Ley 41/2002 de autonomía del paciente, esta explicación tiene como objetivo informarle sobre el servicio de cesación tabáquica que se presta en esta farmacia.

\section{FINALIDAD DE ESTE SERVICIO}

-Que las personas dejen de fumar.

-La individualización, apoyo y acompañamiento al fumador durante todo el proceso.

-El ofrecimiento de educación sanitaria sobre los beneficios y la importancia de dejar de fumar, así como de los perjuicios para el fumador y los que están a su alrededor.

-El aumento de la motivación a los fumadores para que dejen de fumar.

-La provisión de herramientas eficaces para conseguir dejar de fumar a las personas dispuestas a hacer un intento serio.

Este servicio profesional farmacéutico asistencial no sustituye a la visita a su médico cuando esta sea preciso (por ejemplo, cuando empeore su salud).

\section{¿CUÁNDO ESTÁ INDICADO?}

Este servicio está indicado para los fumadores, ofreciéndoles distintas intervenciones dependiendo de su disposición a dejar de fumar:

-Fumadores que no deseen dejar de fumar, se realiza una intervención mínima para concienciar y progresar hacia una etapa más avanzada en su decisión de dejar de fumar.

-Fumadores que deseen dejar de fumar, se les ofrece atención personalizada y seguimiento, proporcionándoles las herramientas para aumentar la probabilidad de lograrlo.

- Fumadores que están dejando de fumar, se les ofrece atención personalizada y seguimiento.

\section{¿EN QUÉ CONSISTE?}

El servicio de cesación tabáquica es un servicio profesional farmacéutico asistencial no gratuito, encaminado a motivar, apoyar y acompañar al fumador en su propósito de dejar de fumar. Se actúa a dos niveles:

-Individualizando sobre las ventajas de abandonar el consumo de tabaco a aquellos fumadores que no se planteen dejar de fumar, mediante una intervención mínima e intentando que cambien de opinión. 
-Ayudando a aquellos fumadores que quieran dejarlo, mediante la realización periódica de entrevistas clínicas entre el paciente y el farmacéutico, en las que éste recoge su historia tabáquica y otros datos como patologías y fármacos que toma, así como proporcionando soporte farmacológico y/o consejos cognitivoconductuales, informando, motivando, monitorizando, interviniendo y ayudando, según sus necesidades, con el fin de conseguir dejar de fumar.

\section{¿DÓNDE SE REALIZA Y CUÁNTO TIEMPO REQUIERE?}

Este servicio se lleva a cabo mediante una entrevista en una zona de atención personalizada de la farmacia que garantice la confidencialidad. La información recabada está sometida al secreto profesional, salvo que el paciente decida que se comparta con su médico u otro profesional sanitario. La duración estimada de la primera visita es de alrededor de 60 minutos. El resto de las visitas de seguimiento que se programan a lo largo de un año serán de alrededor de unos 20 minutos. Una vez terminada la consulta, se le entregará un informe del servicio realizado acompañado de una hoja de información. Si el farmacéutico lo estima conveniente también se le entregará un documento de comunicación con el médico u otros profesionales sanitarios.

\section{¿QUÉ SE NECESITA?}

Pedir una cita en la farmacia para que se le preste el servicio.

\section{¿QUÉ BENEFICIOS SE PUEDEN ESPERAR?}

Con el cese del consumo de tabaco, mejorará en muchos aspectos. Los beneficios asociados a dejar de fumar son tanto a corto como a largo plazo, convirtiéndose en exfumador al año de haber dejado de fumar. Reducirá su riesgo cardiovascular de manera significativa igualándolo al de un no fumador. Recuperará el olfato, aumentará su resistencia física, su pulso se normalizará... en definitiva ganará en calidad y cantidad de vida.

\section{¿QUÉ RIESGOS SE PUEDEN ESPERAR?}

Este servicio profesional no tiene en principio ningún riesgo para usted, ya que cuando la recomendación del farmacéutico consista en la supresión, cambio o variación de dosis de un medicamento de prescripción médica, deberá ponerse en conocimiento de su médico para que to valide su realización antes de hacer efectiva la modificación.

\section{¿QUÉ CONSECUENCIAS PUEDE TENER SU REALIZACIÓN?}

Una mejora de su estado de salud y su bienestar, así como un aumento de su cantidad y calidad de vida. 


\section{EXPLICACIÓN AL PACIENTE DEL SERVICIO DE INDICACIÓN FARMACÉUTICA}

De conformidad con la Ley 41/2002 de autonomía del paciente, esta explicación tiene como objetivo informarle sobre el servicio de indicación farmacéutica que se presta en esta farmacia.

\section{FINALIDAD DE ESTE SERVICIO}

Ofrecerle la indicación más adecuada para el problema de salud (síntoma menor) por el que consulte.

\section{¿CUÁNDO ESTÁ INDICADO?}

Este servicio está dirigido a cualquier paciente que acuda a la farmacia consultando por un problema de salud (síntoma menor) para el que necesite un remedio o solución.

\section{¿EN QUÉ CONSISTE?}

El Servicio de Indicación Farmacéutica es un servicio profesional farmacéutico asistencial que consiste en ofrecer la indicación más adecuada al paciente que consulta por un determinado problema de salud (síntoma menor). Esta indicación puede consistir en recomendaciones sobre el estilo de vida, medicamentos o productos sanitarios que no requieren receta médica, o en la derivación al médico para su evaluación. Para ello, el farmacéutico ha de realizarle una breve entrevista que le permita conocer su situación concreta, por lo que necesitará su colaboración para que le conteste a algunas preguntas sobre dicho problema de salud y aquellos otros que pueda sufrir, sobre su medicación, y posibles alergias.

\section{¿DÓNDE SE REALIZA Y CUÁNTO TIEMPO REQUIERE?}

Este servicio profesional se lleva a cabo mediante una entrevista en una zona de atención personalizada de la farmacia que garantice la intimidad y la confidencialidad. La información recabada está sometida al secreto profesional, salvo que usted decida que se comparta con su médico u otro profesional sanitario. La duración de este servicio depende de su situación concreta, siendo lo más habitual entre 5-10 minutos.

Una vez terminada la consulta, se le entregará un informe del servicio realizado acompañado de una hoja de información. Si el farmacéutico lo estima conveniente también se le entregará un documento de comunicación con el médico u otros profesionales sanitarios. 


\section{¿QUÉ SE NECESITA?}

Acudir a la farmacia para que se le preste el servicio colaborando con el farmacéutico en la entrevista y aportando todos los datos necesarios sobre sus medicamentos y problemas de salud.

\section{¿QUÉ BENEFICIOS SE PUEDEN ESPERAR?}

Obtener la indicación más adecuada para resolver el problema de salud (síntoma menor) por el que consulte, en función de su situación concreta y de la información que usted aporte.

\section{¿QUÉ RIESGOS SE PUEDEN ESPERAR?}

Los riesgos de este servicio están muy limitados para usted, ya que cuando el problema de salud requiera de evaluación médica, el farmacéutico se lo comunicará para que vaya a visitar a su médico. Los riesgos de este servicio son limitados, ya que cuando la recomendación del farmacéutico consista en la supresión, cambio o variación de dosis de medicamentos o tratamientos prescritos por su médico, deberá ponerse en contacto con este último para que to valide su realización antes de hacer efectiva la modificación. En caso de que empeore su salud, póngase en contacto con su médico o acuda a un servicio de urgencias, ya que este servicio no sustituye la asistencia médica cuando ésta sea preciso (por ejemplo, cuando empeore su salud).

\section{¿QUÉ CONSECUENCIAS PUEDE TENER SU REALIZACIÓN?}

Una mejora del problema de salud sobre el que usted consulta. 


\section{EXPLICACIÓN AL PACIENTE DEL SERVICIO DE MEDICIÓN Y CONTROL DE LA PRESIÓN ARTERIAL Y EL RIESGO VASCULAR}

De conformidad con la Ley 41/2002 de autonomía del paciente, esta explicación tiene como objetivo informarle sobre el servicio de medición y control de la presión arterial y el riesgo vascular que se presta en esta farmacia.

\section{FINALIDAD DE ESTE SERVICIO}

Medirle su presión arterial (PA) y, en caso necesario, ayudar a controlarla.

Determinarle su riesgo vascular (RV) y asesorarle e implicarle en su control.

Mejorar su conocimiento sobre los estilos de vida saludables, medidas higiénico-dietéticas y farmacoterapéuticas relacionadas con los factores de RV: hipertensión, dislipemia, diabetes, tabaquismo, obesidad, estrés.

Colaborar con el médico u otros profesionales de la salud que le atiendan en el control de su PA y RV.

\section{¿CUÁNDO ESTÁ INDICADO?}

En principio este servicio está dirigido a cualquier paciente o usuario que desee conocer su PA y su RV. Pero es más útil para aquellos pacientes que presenten al menos un factor de RV: hipertensión, diabetes, dislipemia, tabaquismo, obesidad, estrés.

\section{¿EN QUÉ CONSISTE?}

Este servicio profesional farmacéutico asistencial no es gratuito y consiste en la medida de su PA y el cálculo de su RV, es decir, calcular la probabilidad que usted tiene de sufrir un evento cardiovascular en los próximos 10 años. La medida de la PA se puede efectuar a través de distintos métodos de medición validados en función de sus características y necesidades, y el cálculo del RV también puede realizarse a través de diferentes métodos en función de su edad, su sexo y la presencia de diferentes factores de riesgo. También consiste en prestarle ayuda para que mantenga, controle, reduzca sus valores de PA y mantenga y/o disminuya su RV. Para ello se realiza -en el caso de la PA- el seguimiento de los valores obtenidos respecto de sus objetivos de control y en el caso del RV, el seguimiento del mantenimiento de los diferentes factores de riesgo, así como en ambos casos, el asesoramiento farmacéutico y la colaboración con el resto de los profesionales sanitarios. 


\section{¿DÓNDE SE REALIZA Y CUÁNTO TIEMPO REQUIERE?}

Este servicio profesional se lleva a cabo mediante una entrevista en una zona de atención personalizada de la farmacia que garantice la confidencialidad. La información recabada está sometida al secreto profesional, salvo que usted decida que se comparta con su médico u otro profesional sanitario. La duración de una medida aislada de la PA es de unos 10 minutos en total, 5 minutos de ellos sentados cómodamente en un lugar tranquilo antes de comenzar la medición y los 5 minutos restantes para registrar 2-3 mediciones separadas 1-2 minutos, de forma que la PA es el promedio de las mediciones. En caso de que entre las primeras 2 mediciones haya una diferencia $>$ $10 \mathrm{~mm}$ de mercurio habrá que repetir las medidas. En el caso de las medidas de PA en pacientes con cifras de PA inestables debido a arritmias, como los pacientes con fibrilación auricular (FA), pueden ser necesarias mediciones adicionales y utilizar métodos de auscultación manual, ya que la mayoría de los dispositivos automáticos no están validados para medir la PA de los pacientes con FA. Para la medida de la PA durante todo un día o dos, se requiere que venga el día de antes a la farmacia para programar y colocarle el aparato que la mide y darle unas sencillas instrucciones, $y$, que tras las 24/48 horas de llevar puesto el aparato que le mide la PA, vuelva a la farmacia para poder quitarle el aparato y descargar los datos medidos. En cuanto al cálculo del RV, puede tardar de 5-10 minutos.

Una vez realizada la medición de la PA y el cálculo del RV, se le entregará un informe del servicio realizado acompañado de una hoja de información. Si el farmacéutico lo estima conveniente también le entregará un documento de comunicación con el médico u otros profesionales sanitarios.

\section{¿QUÉ SE NECESITA?}

Pedir una cita en la farmacia para que se le preste el servicio. En el caso de que se vaya a medir la PA es conveniente que no haya tomado bebidas o sustancias estimulantes como el café, té... que no haya practicado ejercicio físico durante las últimos 30 minutos, que no haya tomado el tratamiento para la hipertensión (en caso de que lo tome), que ya haya orinado, y en el caso del cálculo del RV es necesario que nos indique el valor del colesterol total que figura en el informe del análisis de sangre más reciente que le hayan realizado.

\section{¿QUÉ BENEFICIOS SE PUEDEN ESPERAR?}

El conocimiento de los valores de su PA y de su RV, la mejora del conocimiento de los factores de RV y la mejora en la prevención de eventos cardiovasculares. 


\section{¿QUÉ RIESGOS SE PUEDEN ESPERAR?}

Los riesgos de este servicio están muy limitados para usted, ya que tanto la medición de la PA, como la evaluación del RV se llevan a cabo utilizando técnicas o métodos que no implican ningún tipo de daño. Cuando la recomendación del farmacéutico consista en la supresión, cambio o variación de dosis de medicamentos o tratamientos prescritos por su médico, deberá ponerse en contacto con este último para que to valide su realización antes de hacer efectiva la modificación. En caso de que empeore su salud, póngase en contacto con su médico o acuda a un servicio de urgencias, ya que este servicio no sustituye la asistencia médica cuando ésta sea preciso (por ejemplo, cuando empeore su salud).

\section{¿QUÉ CONSECUENCIAS PUEDE TENER SU REALIZACIÓN?}

Una ayuda para mejorar el control de su PA y el conocimiento de su RV, así como para reducir los factores de riesgo que usted puede modificar para prevenir eventos cardiovasculares. 


\author{
ANEXO 5. Hojas de información al paciente del Servicio de revisión de uso \\ de los medicamentos (RUM) de SEFAC e_XPERT ${ }^{\circledR}$
}

\title{
EXPLICACIÓN AL PACIENTE DEL SERVICIO DE REVISIÓN DE USO DE LOS MEDICAMENTOS (RUM)
}

De conformidad con la Ley 41/2002 de autonomía del paciente, esta explicación tiene como objetivo informarle sobre el servicio de revisión de uso de los medicamentos (RUM) que se presta en esta farmacia.

\section{FINALIDAD DE ESTE SERVICIO}

Mejorar el conocimiento, la adherencia y el proceso de uso de los medicamentos que utilice.

\section{¿CUÁNDO ESTÁ INDICADO?}

Se ha observado que hay un gran porcentaje de pacientes que usan mal los medicamentos y esto da lugar a problemas relacionados con los medicamentos o a no obtener todo el beneficio que se debería obtener de éstos. Por ello, en principio este servicio está dirigido a cualquier paciente que utilice medicamentos y desconozca la información mínima para su uso correcto, que al leer el prospecto tenga dudas sobre si tomarlo o no, o preocupaciones sobre los efectos secundarios, que tenga alguna dificultad para cumplir con su tratamiento, haya sufrido recientemente cambios significativos en su tratamiento farmacológico, use regularmente más de 5 medicamentos, o que éstos sean medicamentos complejos de utilizar o precisen de una vigilancia más estrecha.

\section{¿EN QUÉ CONSISTE?}

Este servicio profesional farmacéutico asistencial no es gratuito y consiste en la realización por el farmacéutico junto con usted (o su cuidador en caso necesario) de una revisión estructurada del grado de conocimiento que tiene de sus medicamentos y del uso que hace de los mismos, para ayudarle a emplear sus medicamentos con mayor efectividad, ofreciéndole asesoramiento sobre su utilización correcta, y asegurando que entiende por qué los tiene que tomar y sabe cómo utilizarlos y/o administrarlos, identificando los posibles problemas y realizando las recomendaciones oportunas tanto sobre medidas higiénico-dietéticas y estilos de vida saludables, como sobre los medicamentos que utiliza.

La idea básica del servicio RUM es utilizar los conocimientos de los farmacéuticos para asesorarle en la manera de utilizar correctamente sus medicamentos. 


\section{¿DÓNDE SE REALIZA Y CUÁNTO TIEMPO REQUIERE?}

Este servicio profesional se lleva a cabo mediante una entrevista en una zona de atención personalizada de la farmacia que garantice la confidencialidad. La información recabada está sometida al secreto profesional, salvo que usted decida que se comparta con su médico u otro profesional sanitario. La duración dependerá del número de medicamentos a revisar y de la información que usted ya tenga sobre sus medicamentos. Aproximadamente revisar 6 medicamentos puede tardar alrededor de 30 minutos.

Una vez terminada la revisión de sus medicamentos se le entregará un informe del servicio realizado acompañado de una hoja de información. Si el farmacéutico lo estima conveniente también se le entregará un documento de comunicación con el médico u otros profesionales sanitarios involucrados en los cambios acordados.

\section{¿QUÉ SE NECESITA?}

Pedir una cita en la farmacia para que se le preste este servicio. El día en el que se le vaya a prestar el servicio deberá traer a la farmacia todos los medicamentos que utiliza, así como la hoja de tratamientos, sin olvidarse de otros productos que tome para su salud (plantas medicinales, remedios, etc.).

\section{¿QUÉ BENEFICIOS SE PUEDEN ESPERAR?}

La mejora del conocimiento, del cumplimiento de las tomas y del correcto uso en general de los medicamentos que utilice para su salud.

La detección y resolución de situaciones de mal uso o uso inefectivo de sus medicamentos.

La prevención de la aparición de problemas relacionados con los medicamentos.

La mejora del control de sus problemas de salud y la forma de enfrentarse a los posibles efectos secundarios.

El conocimiento de la información necesaria para resolver sus dudas sobre los medicamentos que toma, para instruirle en su proceso de uso y para facilitar su adherencia al tratamiento.

La reducción del gasto derivado del uso innecesario y del mal uso de los medicamentos, así como disminuir los riesgos medioambientales.

\section{¿QUÉ RIESGOS SE PUEDEN ESPERAR?}

Los riesgos de este servicio son limitados, ya que cuando la recomendación del farmacéutico consista en la supresión, cambio o variación de dosis de medicamentos o tratamientos prescritos por su médico, deberá ponerse en contacto con este último para que valide su realización antes de hacer efectiva la modificación. 
En caso de que empeore su salud, póngase en contacto con su médico o acuda a un servicio de urgencias, ya que este servicio no sustituye la asistencia médica cuando ésta sea preciso (por ejemplo, cuando empeore su salud).

\section{¿QUÉ CONSECUENCIAS PUEDE TENER SU REALIZACIÓN?}

La mejora del uso de los medicamentos que utilice, la mejora de la salud que comportaría ajustar bien la medicación tras la revisión que se lleve a cabo, y una ayuda para prevenir los problemas relacionados con los medicamentos. 


\section{ANEXO 6. Modelo de consentimiento informado para la prestación de SPFA y tratamiento de datos personales de SEFAC e_XPERT ${ }^{\circledR}$}

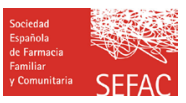

Paseo de las Delicias 31

12 Madrid

Barcelona

TIf: 915221313

info@sefac.org

CONSENTIMIENTO INFORMADO para prestación de servicios profesionales en farmacia comunitaria y tratamiento de datos personales en la aplicación web denominada SEFAC e_XPERT ${ }^{\circledast}$.

D./Da. con DNI

y domicilio en

\section{DECLARA QUE:}

Que he sido informado por el farmacéutico de las ventajas para mi salud de recibir el servicio profesional que presta esta farmacia denominado

como de sus objetivos, funcionamiento y compromisos que conlleva, todo ello detallado en el documento anexo "Explicación del servicio profesional". Igualmente, se me ha informado del precio del servicio, el horario en que se prestará, etc., y de la posibilidad que tengo de revocar este consentimiento en cualquier momento, sin que ello suponga ningún menoscabo de mi atención farmacéutica.

Que estoy conforme y consiento recibir el citado servicio profesional y que, para dicho fin, se registren y traten por el farmacéutico mis datos personales y clínicos a través de la aplicación web SEFAC e_XPERT .

Adicionalmente, se me ha comunicado la posibilidad opcional de beneficiarme de la aplicación móvil denominada PATIENT e_XPERT ${ }^{\oplus}$, explicándome sus ventajas, objetivo, funcionamiento y compromisos que conlleva, que se contienen en el documento anexo "Explicación de la App". Sin perjuicio de que puedo tomar mi decisión más adelante, manifiesto ahora que:

$\square$ Acepto la utilización de esta herramienta.

No acepto de momento la utilización de esta herramienta.

He sido advertido de que los datos personales, incluidos los de salud, que facilite serán tratados con el fin exclusivo de prestarme el citado servicio profesional y que se conservarán mientras sean necesarios para mi asistencia. De acuerdo con lo previsto en el art. 13 del Reglamento 2016/679 europeo de protección de datos (RGPD), he sido informado de que puedo ejercitar gratuitamente mis derechos de acceso, rectificación, cancelación, oposición, portabilidad, limitación del tratamiento y olvido, dirigiéndome por escrito a la farmacia, bien acudiendo presencialmente, bien por correo postal o electrónico en la dirección info@sefac.org. Además, se me ha comunicado que me asiste el derecho a presentar una reclamación ante la Agencia Española de Protección de Datos, si fuera el caso.

Igualmente, acepto que la información recabada durante la realización del servicio que se me preste, siempre previamente anonimizada, sea cedida con fines estadísticos y de mejora del servicio a la Sociedad Española de Farmacia Familiar y Comunitaria (SEFAC), con sede en Madrid, Paseo de las Delicias, 31 - Esc. Izq. 40 Dcha. (28045). También estoy conforme que la citada información anónima pueda ser utilizada por SEFAC con fines de docencia e investigación, cumpliendo las 
previsiones de la Ley Orgánica 3/2018, de 5 de diciembre, de Protección de Datos Personales y garantía de los derechos digitales, y demás normativa de aplicación. La cesión de datos referida en este párrafo excluye expresamente el volcado automático de los datos procedentes de las recetas y de su proceso de facturación, debiéndose respetar en este sentido las limitaciones que imponga el concierto farmacéutico convenido con el Servicio Público de Salud.

Por lo que se refiere a la información obtenida por la prestación del servicio, solo se cederá a otro profesional sanitario (por ejemplo, el médico) con mi consentimiento.

Por último, para el supuesto de cambio en la titularidad de la farmacia, acepto que el nuevo titular o titulares puedan seguir prestándome el servicio y tratando mis datos personales, mientras no disponga otra cosa.

En

Fdo.: El farmacéutico a .......... de

de

Fdo.: El usuario 


\title{
ANEX0 7. Anexo del consentimiento informado para la actualización del consentimiento sobre la APP “PATIENT e_XPERT ${ }^{\circledR}$ "
}

\author{
ANEXO PARA ACTUALIZACIÓN DE CONSENTIMIENTO SOBRE LA \\ APP “PATIENT E_XPERT ${ }^{\circledR ”}$
}

Respecto de la aplicación móvil denominada PATIENT e_XPERT ${ }^{\circ}$, de la que se me han explicado sus ventajas, objetivo, funcionamiento y compromisos que conlleva (que se contienen en el documento anexo "Explicación de la App", que se me ha facilitado), deseo actualizar mi opción inicial en el sentido siguiente:

Acepto la utilización de esta herramienta.

Ceso en la utilización de esta herramienta.

En a .......... de .. de......

Fdo.: El farmacéutico

Fdo.: El usuario 
ANEXO 8. Anexo del consentimiento informado para revocación del consentimiento informado para la prestación de SPFA de SEFAC e_XPERT ${ }^{\circledR}$

ANEXO PARA REVOCACIÓN DEL CONSENTIMIENTO

Revoco mi consentimiento para seguir recibiendo el servicio profesional farmacéutico denominado

En ......................................................, a ........ de ................... de ........

Fdo. El farmacéutico

Fdo. El usuario 